\title{
Original article: \\ Trial of labor after previous cesarean delivery (TOLAC) and association of BMI and previous vaginal delivery with frequency of VBAC Tasleem $H^{l}$, Ghazanfar $H^{2}$
}

\begin{abstract}
$\underline{\text { Abstract }}$
Objectives: To determine the frequency of successful Vaginal Birth after One Cesarean Section in our tertiary care institution and to determine the causes of its failure. Material and Methods: This study was conducted in department of Obstetrics and Gynecology of Shifa International Hospital and Shifa Foundation Community Health Centre Islamabad Pakistan from Feb 2011 to Dec 2014. This study included 592 patients who presented in labor room emergency reception of Obs/Gynae department at term with previous one scar having fulfilled the laid down inclusion criteria for VBAC during ante-natal care. The patients were admitted in hospital and were allowed to proceed for spontaneous labor under vigilant monitoring on complications of trial of scar. Immediate emergency cesarean sections were performed, where indicated. Results: Out of 592 patients $70.7 \%$ were delivered vaginally after previous one cesarean section and $29.3 \%$ had emergency cesarean section. Leading indications for repeat cesarean section was fetal distress, failure to progress and scar tenderness. No maternal and fetal complication occurred in our study. The success rate of Trial of labor after one previous cesarean delivery was lower in obese $(64.38 \%)$ as compared to non-obese women $(82.06 \%)(\mathrm{p}<0.001)$. Women with previous successful vaginal delivery had a success rate of $88.2 \%$ compared with $62.25 \%$ in women without such a history (OR 4.4; 95\% CI 2.7-7.2 p <0.001). Conclusion: Vaginal birth after one lower segment cesarean section should be encouraged with vigilant monitoring provided no obstetric contra-indication to vaginal birth exists.
\end{abstract}

Keywords: (VBAC) Vaginal birth after cesarean section; trial of labor after previous cesarean delivery (TOLAC); uterine rupture, uterine dehiscence; lower segment cesarean section (LSCS).

Bangladesh Journal of Medical Science Vol. 15 No. 04 October'16

\section{Introduction}

The term cesarean delivery is a surgical procedure to deliver a baby through an incision in the uterus. Over the time the Cesarean delivery rate has significantly increase over the time from $18.2 \%$ in 2002 to $30.3 \%$ in $2012^{1}$. It is one of the most common obstetric surgeries performed all over the world ${ }^{2}$. A recent study found out that 69 countries out of a total of 137 exceeded the $15 \%$ threshold recommend by World Health Organization $(\mathrm{WHO})^{3}$. The catch aphorism "Once a cesarean, always a cesarean" came from a paper published in 1916, entitled 'Conservatism in Obstetrics'4. It was neither a prescription nor a recommendation but rather an observation and a caution to avoid a primary cesarean if at all possible, because it might doom the woman to surgical delivery in her future pregnancies. Caesarean sections were usually done for severe cephalo-pelvic disproportion and the classical incision in the muscular body of the uterus was almost universally used $^{5}$.

Medicine has advanced tremendously since $\mathrm{Mr}$. Edwin B. Cragin made this statement in 1916, but

1. Dr. Huma Tasleem, Associate professor, Obs/Gyn, Shifa College of Medicine, Islamabad, Pakistan, E-mail: humaafsar@hotmail.com

2. Dr Haider Ghazanfar, House officer Shifa International Hospital, Islamabad, Pakistan, House Number 19, Askari Villas Chaklala Scheme 3, Rawalpindi, Pakistan.

Corresponds to: Dr Haider Ghazanfar, House officer, Shifa International Hospital, Islamabad, Pakistan, House Number 19, Askari Villas Chaklala Scheme 3, Rawalpindi, Pakistan., E Mail:Haidergh@gmail.com 
the question remains unanswered as to which route of delivery should be taken by a woman with a previous cesarean section. This uncertainty continues to contribute to the United States, having one of the highest cesarean delivery rates of $28.1 \%^{5-7}$. Women with one LSCS have two choices for future birth, that is VBAC (vaginal birth) and RCS (repeat C section). The mode of delivery of second baby after first LSCS is very important, as VBAC after 2 is still not practicing in many parts of the world especially in underdeveloped or less developed countries so the VBAC after one LSCS become very important in such areas. In some part of the world; a very small numbers of Obstetricians are giving trial of labor to previous one scar. The aim of this study was to determine the frequency of successful vaginal birth after one Cesarean section in our tertiary care institution and to determine the causes of its failure.

\section{Materials and Method}

This study was carried out at Obstetrics and Gynaecology Department of Shifa International Hospital and Shifa Foundation Community Health Centre, having tertiary care facilities, from Feb 2011 to Dec 2014. Ethical approval for this study was obtained from Institutional Review board and ethical committee of Shifa College of Medicine/ Shifa International Hospital, Islamabad, Pakistan (IRB \# 29-2010). Subjects were carefully selected, keeping in view the inclusion criteria that is patients consent, patients with previous one lower segment cesarean section at term pregnancy (between 37 to 42 completed weeks of gestation), with singleton pregnancy, longitudinal lie and cephalic presentation, patients with estimated fetal birth weight (EFBW) between 2.5 to $3.5 \mathrm{~kg}$ estimated clinically and proved by ultra-sonography. All the patients with Placenta Previa, borderline pelvis, gross cephalo-pelvic disproportion, patients with extended myomectomy scar twin gestation, patients with poly-hydramnios or oligohydramnios and patients with Intra-Uterine Growth Restriction (IUGR) were excluded. Patients with uncontrolled known Diabetes Mellitus, Gestational Diabetes Mellitus (GDM), Pregnancy Induced Hypertension (PIH) and other medical disorders in which VBAC is contra-indicated were also excluded. Patients were only being included after informed written consent.

Initial assessment was carried out by taking a detailed history with stress upon indication of previous cesarean section, type of scar, maternal age, BMI, interval between two pregnancies, history of post cesarean section sepsis and gestational age followed by detailed relevant physical examination. Baseline investigations were done and Obstetric Ultra Sono Graphy (USG) was performed at the emergency reception for fetal well being. All patients were then allowed to proceed for spontaneous labor. An abdominal and pelvic examination was done in every patient and findings were plotted on partogram. During their stay in labor room, scar tenderness and continuous cardiotocography (CTG) in all patients was monitored with great care and as per protocol for High Risk Pregnancy. Analgesia in the form of epidural was offered to every patient and was given on her choice. Instruments were applied as per protocol on indication. Failure to progress was diagnosed in patients when there was no progress in Bishop Score after being in labor for 4 hours despite regular and painful uterine contraction. Fetal distress was labeled if either grade 2 or more meconium was present or there was 3 or more variable or type 2 decelerations present on 20 minutes CTG strip. Scar tenderness was labeled in patients when tenderness present on palpation over uterine scar area in relaxation phase with unfocussed patient's attention. In case of failure of VBAC, immediate cesarean section was performed as per protocol. All findings were recorded on 'Study Proforma'.

After delivery, patients were kept in labor room recovery, for monitoring of pulse, blood pressure, uterine fundus retraction, post partum hemorrhage and urinary output. During this time, baby was stayed along with mother and breast fed. Further management was customized to individual cases. Patients were followed till the time of discharge and follow up visit was advised after one week in our OPD. All data was collected with the help of self-made study Proforma. Data was entered and analyzed, using SPSS version 21.0. For continuous variables (age, BMI, gravida, gestational age), Mean and 'Standard Deviation (SD)' was calculated. For categorical data (mode of delivery, reason for failure of VBAC) frequencies and percentages were calculated. Chi square test was applied to assess the association of BMI with success rate of Trial of labour after previous $\mathrm{C}$-section. A p-value of less $<0.05$ was consider significant. Odds ratio was calculated to assess association between previous vaginal deliveries with successful vaginal birth after Cesarean section.

\section{Results:}

A total of 592 pregnancies which fulfilled the selection criteria were included in this study and were allowed to proceed for vaginal birth after one 
cesarean section. All the enrolled patients were studied and there was no missing value. Mean $( \pm \mathrm{SD})$ patient's age (yrs) and gestational age (wks) was $29.2 \pm 1.08$ and $38.7 \pm 1.04$ respectively. Out of these 592 patients, $400(67.4 \%)$ were on second gravida. Of the 592 cases $418(70.7 \%)$ delivered vaginally after previous one cesarean section and $174(29.3 \%)$ had emergency cesarean section. Among 418 patients who delivered vaginally $366(87.69 \%)$ patients had BMI less than 30 . Out of 174 patients who had emergency cesarean section 94 (54\%) had BMI $\geq 30$. About 244 $(41.3 \%)$ had normal vaginal births, outlet forceps were applied on $90(15.2 \%)$ cases and ventouse delivery was conducted in $84(14.2 \%)$ patients. Out of 174 patients who landed up on cesarean section 78 $(13 \%)$ instances had fetal distress, 58 (9.8\%) patients showed failure to progress. Cesarean section due to scar tenderness was carried out in $38(6.5 \%)$ of the patients. Of the 38 patients $8(21 \%)$ developed scar dehiscence. There was no uterine scar rupture and no maternal or fetal mortality in our study.

The success rate of trial of labor after one previous cesarean delivery was lower in obese $(\mathrm{BMI} \geq 30)$ $(64.38 \%)$ as compared to non-obese women $(\mathrm{BMI}<30)$ $(82.06 \%)(p<0.001)$. Women with previous successful vaginal delivery had a success rate of $88.2 \%$ compared with $62.25 \%$ in women without such a history (OR $4.4 ; 95 \%$ CI 2.7-7.2, $\mathrm{p}<0.001)$.

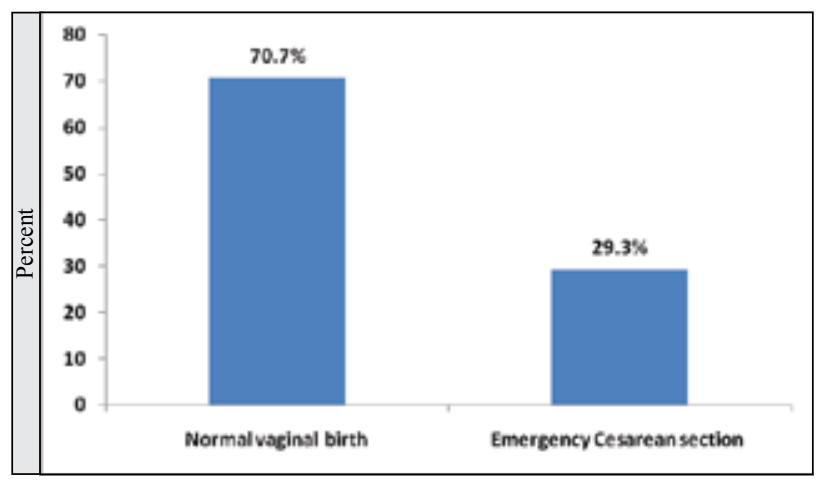

Figure 1: Mode of Delivery after one Cesarean section

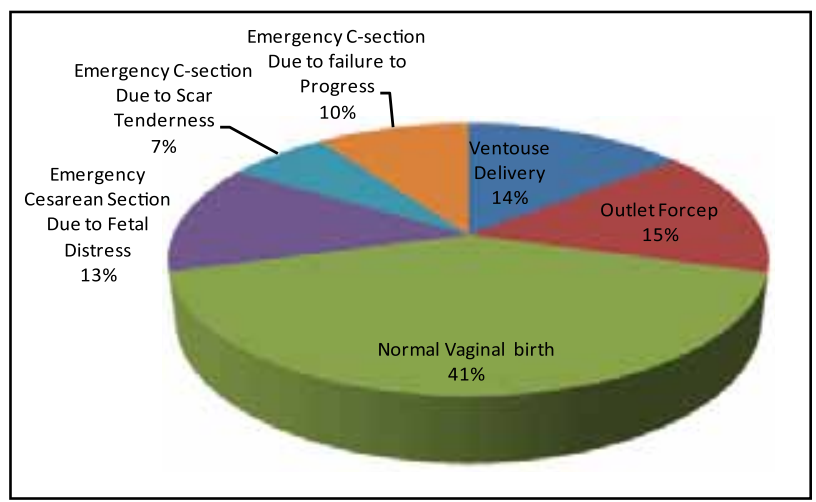

Figure 2: Further Break Down of Mode of Delivery

\section{Discussion:}

Trial of labor after previous cesarean delivery (TOLAC) provides women who wish to have vaginal birth after previous Lower Segment caesarean Section (VBAC). Assessment of Individual risk and chance of success is an important factor in determining appropriate candidates for Trial of Labor after previous Cesarean Delivery ${ }^{8}$. In our study we excluded the patients who were not good candidates for TOLAC. There are many factors in TOLAC causing failure of VBAC and increase in maternal and perinatal morbidity. VBAC is a safe and appropriate choice for most women with previous one LSCS and for some women with previous two LSCS. Current Literature also recommends trial of scar in twin pregnancies and postdates pregnancies ${ }^{9}$. We have excluded twin gestation in our study.

The increase in rate all over the world could be because of more use of cardiotocography (CTG), less use of forceps delivery and no vaginal delivery of breech presentations ${ }^{10-12}$. The practice of repeat cesarean section nonetheless exerts a major influence on the overall increase in cesarean section rate ${ }^{13-14}$. A recent study done in Switzerland found out that rate of Cesarean section had significantly increased from $0.3 \%$ to $1.2 \%$ over a span of 6 years ${ }^{14}$. In this study, we assessed prospectively the frequency of VBAC in 592 patients. The frequency of VBAC was found to be $70.7 \%$.Other studies found the probability of successful VBAC between 60 to $84 \%{ }^{15-18}$.

A number of risk factors for unsuccessful VBAC are mentioned like induced labor, no previous vaginal birth, body-mass index greater than 30 , and previous cesarean section for dystocia ${ }^{19-23}$. A study done on Fourteen thousand five hundred twenty-nine women found out that overall Trial of Labour after one previous cesarean section success rate in obese women $(\mathrm{BMI} \geq 30)$ was lower $(68.4 \%)$ than in nonobese women $(\mathrm{BMI}<30)^{24}$. Similar results were seen in our study.

Numerous other factors associated with decreased likelihood of successful VBAC rate are birth weight greater than $4000 \mathrm{~g}$, previous pre-term cesarean birth, cervical dilatation of less than $4 \mathrm{~cm}$ at the time of admission, station of vertex -2 or higher at the time of admission, less than 2 years from previous cesarean birth, advanced maternal age, non-white ethnicity and short stature ${ }^{25-27}$. Where relevant to the patient's circumstances, these information should be shared during ante-natal counseling process to enable the woman to make the best informed choice. Previous 
vaginal birth is associated with an appropriately 87 to $90 \%$ planned VBAC success rate ${ }^{28-30}$. In our study women with previous successful vaginal delivery had a success rate of $88.2 \%$.

\section{Conclusion:}

In the management of patients with previous cesarean section, regular and intensive ante-natal surveillance is required. Careful observation throughout labor in a well-equipped unit is necessary. Thus proper counseling for trial of labor and evaluation of the cases of women with prior cesarean section is considered a key method of reducing cesarean section rate. Majority of Cases can be with previous one Cesarean section done for non-recurrent indication can be successfully and safely delivered by the vaginal route. There is no doubt that a trial of labor is relatively safe procedure but it is not risk free and should not be undertaken in casual manner.

Conflict of interest: None declared

\section{References:}

1. Al Rifai R. Rising cesarean deliveries among apparently low-risk mothers at university teaching hospitals in Jordan: analysis of population survey data, 20022012Glob Health Sci Pract 2014 13; 2: 195-209.

2. Subedi S. Rising rate of cesarean section - a year review. J Nobel Med Coll. 2012; 2: 72-76.

3. Gibbons L, Belizan JM, Lauer JA, Betran AP, Merialdi $\mathrm{M}$, Althabe $\mathrm{F}$. Inequities in the use of cesarean section deliveries in the world. Am J Obstet Gynecol. 2012; 206: 331.e1-19.

4. Cragin EB. Conservatism in Obstetrics. NY Med J 1916; 104: 1-3.

5. Martin JA, Hamilton BE, Sutton PD, Ventura SJ, Menacker F, Kirmayer S. Births: Final data for 2004. Natl Vital Stat Rep 2006; 55: 1-101.

6. Usha-Kiran TS, Jayawickrama NS. Who is responsible for the rising cesarean section rate? J Obstet Gynaecol 2002; 22: 363-65.

7. Martin JA, Hamilton BE, Osterman MJ. Births in the United States, 2013. NCHS Data Brief 2014; 175: 1-8.

8. American College of Obstetricians and Gynecologists. ACOG Practice bulletin no. 115: Vaginal birth after previous cesarean delivery. Obstet Gynecol. 2010; 116 (2 Pt 1): 450-63.

9. Practice Bulletin No.115: Vaginal birth after previous cesarean section, Clinical management guidelines for
Obstetrician-Gynecologist. August 2010; 116: 450-463.

10. Clark SL, Hankins GD. Temporal and demographic trends in cerebral palsy-fact and fiction. Am J Obstet Gyneco 2003; 188: 628-33.

11. Landon MB,Hauth JC, Leveno KJ, Spong CY, Leindecker S, Varner MW, et al. Maternal and peri-natal outcomes associated with a trial of labour after prior cesarean delivery. N Eng J Med 2004; 351: 2581-89.

12. Oetzinger KR, Macones GA. Operative Vaginal delivery: current trends in obstetrics. Women Health 2008; 4; 281-90.

13. Menacker F. Trends in cesarean rates for first births and repeat cesarean rates for low risk women. United States, 1990-2003. Natl Vital Stat Rep 2005; 54:1-8.

14. Kottmel A1, Hoesli I, Traub R, Urech C, Huang D, Leeners $\mathrm{B}$ et al. Maternal request: a reason for rising rates of cesarean section? Arch Gynecol Obstet. 2012; 286: $93-8$

15. Yeh J, Wackawski WJ, Shelton JA, Reschke J. Temporal trends in the rates of trial of labour in low risk pregnancies and their impact on the rates and success of vaginal birth after cesarean delivery. Am J Obstet Gynaecol 2006; 194: 144-46.

16. Smith GC, White IR, Pell JP, DobbieR. Prediting cesarean section among women attempting vaginal birth after prior cesarean section. PLoS Med 2005; 2: 871-78.

17. Balachandran L, Vaswani PR, Mogotlane R. Pregnancy outcome in women with previous one cesarean section. 
J Clin Diagn Res. 2014; 8: 99-102.

18. Metz TD, Stoddard GJ, Henry E, Jackson M, Holmgren C, Esplin S. Simple, validated vaginal birth after cesarean delivery prediction model for use at the time of admission. Obstet Gynecol. 2013; 122: 571-8

19. Dodd J, Crowther C. Induction of labour for women with previous cesarean birth: A systemic review of the literature. Aust NZ J Obstet Gynaecol 2004; 44: 392-95.

20. Dinsmoor MJ, Brock EL. Predicting failed trial of labour after primary cesarean delivery. Obstet Gynaecol 2004; 103: 282-86.

21. Hibbard JU, Gillbert S, Landon MB, Hauth JC, LevenoKJ, Spong CY,et al. Trial of Labour or repeat cesarean delivery in women with morbid obesity and previous cesarean delivery. Obstet Gynaecol 2006; 108: $125-33$.

22. Peaceman AM, Gersnoviez R, Landon MB, Spong CY, Leveno KJ, Varner MW, et al. The MFMU cesarean registry: Trial of labour for patients with previous cesarean for dystocia. Am J Obstet Gynaecol 2006; 195:1127-31.

23. Tessmer-Tuck JA, El-Nashar SA, Racek AR, Lohse CM, Famuyide AO, Wick MJ. Predicting vaginal birth after cesarean section: a cohort study. Gy st 2014; 77: 121-6. necol Obstet Inve

24. Landon MB, Leindecker S, Spong CY, Hauth JC, Bloom
S, Varner MW, et al. The MFMU cesarean registry: factors affecting the success of trial of labor after previous cesarean delivery. Am J Obstet Gynaecol 2005; 193: $1016-23$

25. Elkousy MA, Sammel M, Stevens EJ, PeripertJF, Macones GA. The effect of birth weight on vaginal birth after cesarean delivery success rates. Am J Obstet Gynaecol 2003; 188: 824-30.

26. Hollard AL, Wing DA, Chung JH, Rumney PJ, Saul L, Nagotte MP,et al. Ethnic disparity in the success of vaginal birth after cesarean delivery. J Matern Fetal Neonatal Med 2006; 19: 483-87.

27. Siddiqui SA. Obstetric factors for unsuccessful trial of labor in second-order birth following previous cesarean. Ann Saudi Med 2013; 33: 356-62.

28. Vidyadhar B Bangal, Purushottam A Giri, Kunaal K Shinde, Satyajit P Gavhane. Vaginal Birth after Cesarean Section. N Am J Med Sci 2013; 5: 140-144.

29. Shaheen N, Khalil S, Iftikhar P. Prediction of successful trial of labour in patients with a previous caesarean section. J Pak Med Assoc 2014; 64: 542-5.

30. Obeidat N, Meri ZB, Obeidat M, Khader Y, Al-Khateeb $\mathrm{M}$, Zayed F, et al. Vaginal birth after caesarean section (VBAC) in women with spontaneous labour: predictors of success. J Obstet Gynaecol 2013; 33: 474-8. 